\title{
OBSERVATORIO DE PROBLEMÁTICAS DE DISEÑO DE INFORMACIÓN EN EL ENTORNO CIUDAD
}

\section{Entorno Ciudad}

Nidia Raquel Gualdrón Cantor

nrgualdronc@libertadores.edu.co Fundación Universitaria Los Libertadores. Comunicación, cultura y tecnología. Bogotá, Cundinamarca. 
Nadie sabe todo, todos saben algo. Pellegrini (2004)

\section{Resumen}

a ciudad es un territorio espacio-temporal que, por la estructura de relaciones entre las

— personas con los entornos, los artefactos y los servicios, se configura como un sistema complejo en constante cambio. Con frecuencia los habitantes enfrentan situaciones cotidianas en las que la ausencia, insuficiencia o confusión en la información relativa a éstos, obstaculiza esas relaciones o las carga de experiencias negativas en tanto implican para los usuarios un mayor esfuerzo cognitivo, en tiempo o en el simple disfrute de los mismos. El interés de los diseñadores en formación, acerca de los fallos de diseño de información (DI), que por múltiples razones se repiten como patrones en diferentes entornos de la ciudad, lleva a proponer un Observatorio como vector basado en la inteligencia colectiva que enlace a la industria (arquitectura, ingeniería, diseño urbano, diseño de servicios), la academia y la política pública en la generación de sistemas de información que contemplen la interrelación existente entre las diferentes variables y que sirvan como insumo en los procesos de validación de los productos de DI para los diferentes entornos, servicios, interfaces y artefactos que generan interacción en la ciudad. Aquí se presenta la prospectiva de configuración del Observatorio en una ruta de trabajo por etapas, así como los avances y hallazgos en la delimitación del universo de observación, la exploración de métodos de diseño para la recolección y categorización de información, y las instancias para considerar en el diseño del sistema de información. En el sector productivo, los alcances de mayor interés pueden estar en la infraestructura o el diseño de los artefactos; no obstante, la etapa del diseño de información que facilita o guía su uso con frecuencia ocurre al final, cuando se dispone de menos tiempo para su testeo o se le confiere menor importancia.

\section{Introducción}

El proyecto de Observatorio que ha emprendido el semillero en Diseño de Información, se propuso identificar situaciones en las que se evidenciaban fallos en el DI, que afectan la interacción persona/espacio físico, persona/servicio, persona/artefacto y persona/interfaz en el entorno ciudad, con el fin de configurar un sistema de información como insumo y como instrumento para la investigación aplicada a potenciales proyectos de diseño que vinculen a la academia, la industria y la política pública, que contemplen la interrelación existente entre las diferentes variables, y que aporten a los procesos de validación de los artefactos de DI.

¿Por qué un observatorio? Por parecer menores un sinnúmero de problemáticas de DI, son subordinadas en desarrollos arquitectónicos, ingenieriles, civiles, tecnológicos, industriales y de servicios, entre otros. Identificarlas tiene valor cuando se hace por medio de una estrategia colaborativa que abre la participación a un mayor número de agentes que, en un ambiente propicio, dan valor agregado a datos, con el fin de activar el potencial de los artefactos de diseño. Un observatorio es un conjunto de actividades y procedimientos 
destinados a identificar y evaluar aquella información que se produce en un contexto, como mecanismo para generar evidencia útil para la toma de decisiones en un ámbito de competencia, en este caso, el DI. En términos de Angulo (2009), un catalizador de la inteligencia colectiva, en el que, como lo expresa Pellegrini (2004), se enfatiza el hecho de que nadie sabe todo y todos saben algo.

Según la naturaleza y el ámbito de interés del observatorio, sus funciones pueden ser una o varias de las siguientes: captación y análisis de información sobre el fenómeno de observación, desarrollo de métodos de recolección, análisis, procesamiento y sistematización de la información, investigación -revisión, descripción, caracterización, evaluación, discusión, cuestionamiento-de los datos y hallazgos que aparecen en el espacio de observación pertinente a su área de interés, monitoreo, generación de indicadores para conocer y medir el fenómeno observado, difusión a fin de facilitar la toma de decisiones políticas y el desarrollo de planes y programas, así como intercambio y colaboración en la gestión de información. Los resultados de esta observación estratégica, al ser transformados en conocimiento revelan aspectos inéditos de la experiencia cotidiana en la ciudad, que se constituyen en insumos para la investigación en diseño -en este caso-, para el apoyo en la toma de decisiones en política pública y para la formulación de escenarios a futuro (Angulo, 2009).

\section{Metodología}

Luego de preguntarse por el estado del arte de los observatorios en diseño, con respuestas aún incipientes, se ha esbozado en prospectiva la ruta de trabajo para la configuración del Observatorio de problemáticas de diseño de información (OPDI), contemplando las siguientes etapas:

\section{- 1. Definición del universo de observación ¿Qué observar?}

El diseño de información tiene como objetivo hacer inteligible el mundo circundante, mediante el facilitar los procesos de percepción, lectura, comprensión, memorización y uso de la información presentada. El buen diseño de información invita a ser usado, reduce cansancio y errores en el procesamiento de la información (esfuerzo cognitivo) y optimiza el tiempo (Frascara, 2011).

A menudo se presentan falencias en estos procesos, en la relación de los usuarios con entornos, interfaces, artefactos e, inclusive, en la interacción persona a persona. Estos pueden ser identificados, pero, también, medidos objetivamente en conductas observables como la cantidad de información recordada, la exactitud de la información recordada, el tiempo necesario para encontrar información en un documento, para llenar un formulario, entre otras.

En ese amplio universo, el primer desafío fue determinar las categorías de observación, para lo cual se utilizó la técnica cardsorting (Ideo.org; Usability.gov, s.f.), en varias instancias, a saber: 
1. En método presencial abierto: en 8 grupos de $4-5$ estudiantes, se rotaron tarjetas con las preguntas que se muestran en la figura 1 , a las cuales los participantes respondieron libremente en tarjetas suministradas para la actividad.

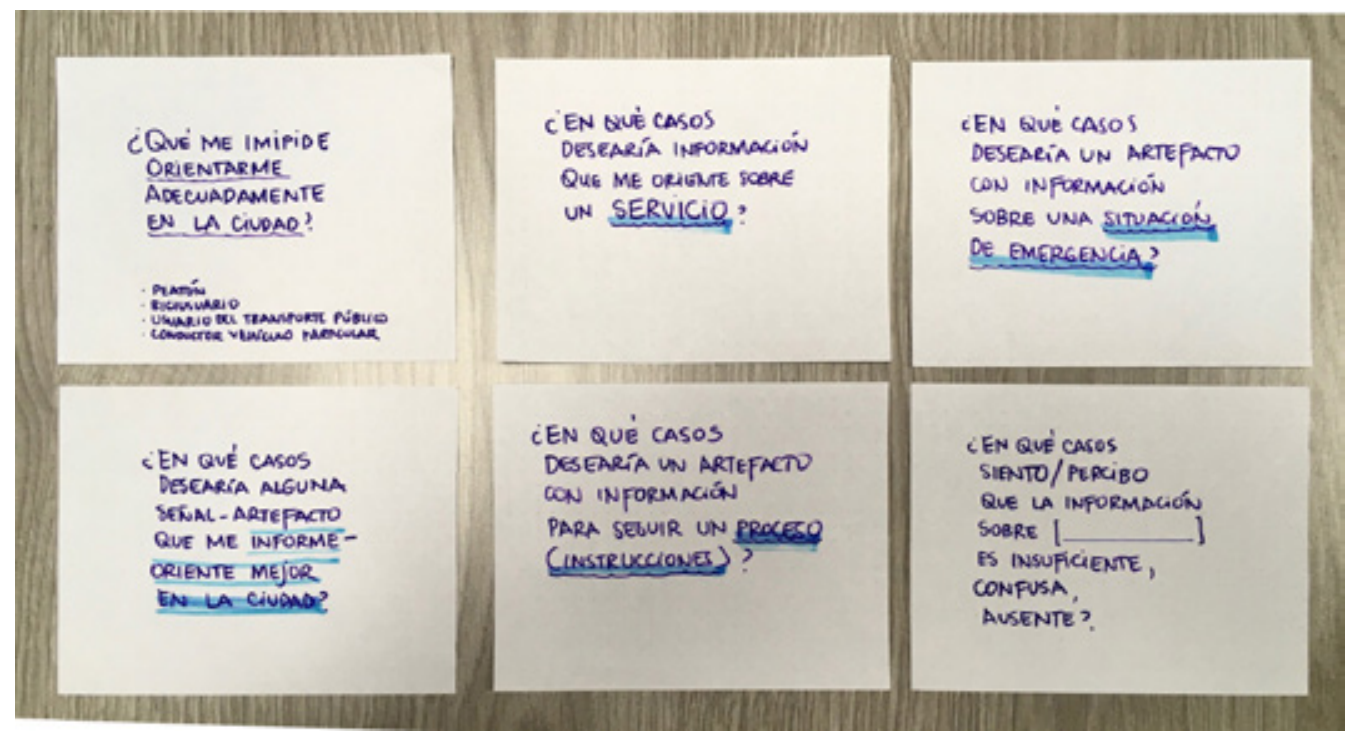

Figura 1. Tarjetas detonantes del universo de observación Fuente: elaboración propia.

2. En método presencial híbrido: en tres sesiones con grupos diferentes de 3-4 personas (estudiantes y funcionarios), se rotaron tarjetas con las categorías que siguen a continuación, en las cuales los participantes escribieron o dibujaron situaciones relacionadas:

En qué casos percibe usted ausente, insuficiente o confuso el cómo se presenta la información que necesitan los usuarios en situaciones concretas, para:

- Orientarse -llegar, ubicarse, recorrer, salir de un lugar-.

- Saber sobre un servicio -procesos, horarios, costos, tiempos, a quién dirigirse, otros-.

- Saber sobre normativa, regulaciones y situaciones de peligro.

- Seguir instrucciones - proceso paso a paso, armado de artefactos, preparación de medicamentos o recetas, cómo llegar a un lugar-.

- Memorizar y usar información -sucesos, hechos, rutas, información relevante con propósitos fijos-.

Con estos insumos, se procedió a la elaboración colectiva de un gigamap para visualizar la información obtenida y analizar sus relaciones (véase figura 2). El gigamapping, visualización multipropósito y multicapa desarrollada ampliamente por Sevaldson (2011), es un dispositivo de mapeo para el diseño, que tiene entre sus usos e intencionalidades: a) comprender la complejidad: el sistema, sus subsistemas y supra-sistemas, su entorno y su paisaje; b) diseñar, compartir, alinear y criticar una imagen de una situación compleja; 
c) comprender y compartir los campos problemáticos (problemáticas); d) modular la pertinencia y priorizar la importancia; y e) facilitar la crítica y ajuste de límites.

Un gigamap está intencionalmente en construcción. Su pertinencia en este ejercicio, está dada no sólo por la visualización en simultaneo de las relaciones generadas entre las categorías, sino por la potencia del análisis Zoom, Innovación, Potencial (ZIP), que sirve para encontrar áreas potenciales para intervenciones e innovaciones. El Zoom invita a marcar áreas o puntos en el mapa, que necesitan más investigación; la I de idea -intervención o innovación-, señala acciones no necesariamente nuevas, pero que sí modifican y cambian el sistema; y $\mathrm{P}$ significa potencial, problema o problemática: si hay un problema hay un potencial de mejora. Este análisis puede darse durante el desarrollo del mapa o en sesiones posteriores que generen interés de investigación sobre un área específica.

Autores del Instituto Nacional de Diseño en India, en el Visualizing Complex System (RSD7, 2019), presentaron casos de estudio en los que el gigamap sirvió como herramienta no sólo para mapear casos como el acceso a la electricidad en India, el diseño de un sistema para mejorar la vida de los trabajadores de las panaderías, el rediseño de un sistema de residuos para la eliminación de residuos urbanos, entre otros, sino como área de juego segura para probar nuevas ideas y debatir oportunidades, para crear, así, un terreno de deliberación sobre los contextos revelados para prever soluciones centradas en las personas y planificar una ejecución efectiva.

De esta manera, del registro en el gigamap elaborado, los participantes decantaron cuatro categorías de observación de problemáticas en diseño de información, presentes en la interacción con la ciudad:

c. Persona/ espacio físico: relativa a lugares de recorrido por la ciudad, de prestación de servicios, de carácter habitacional, laboral, comercial o de ocio, en los que es ausente, insuficiente o confuso el sistema de orientación y las regulaciones normativas o de seguridad.

d. Persona/ servicio: como los de orden hospitalario, trámites de servicios públicos, gestión ante entidades públicas o privadas, transporte, entre otros, que impliquen instrucciones paso a paso, información sobre horarios, tarifas restricciones, prevención de re-procesos y otros.

e. Persona/interfaz: los mapas de rutas del sistema de transporte masivo, el uso de máquinas de recarga de tarjetas, cajeros electrónicos, mapas de información, inclusión de braille, la simple señal de hale o empuje en las puertas; es decir, interfaces de imagen fija o en movimiento que prescinden de la mediación humana para suministrar determinada información o realizar un proceso.

f. Persona/ artefacto: se refiere a la interacción con objetos que tienen algún grado de novedad para el usuario en tanto a su utilización, como los gimnasios al aire libre, determinados mecanismos de ingreso a edificios, objetos para armar, entre otros. 

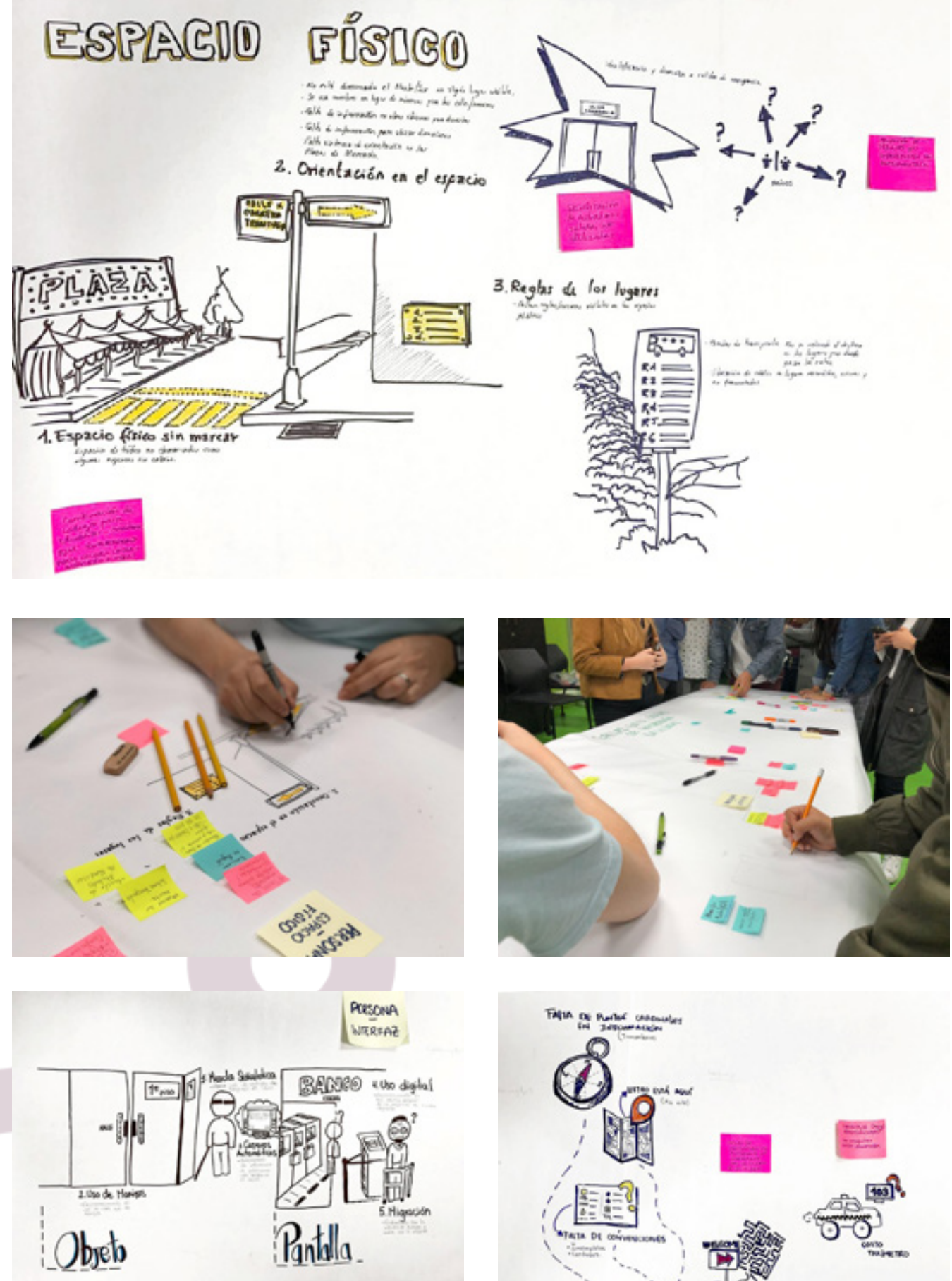

Figura 2. Gigamap que registra la primera fase: universo de observación

Fuente: elaboración propia.

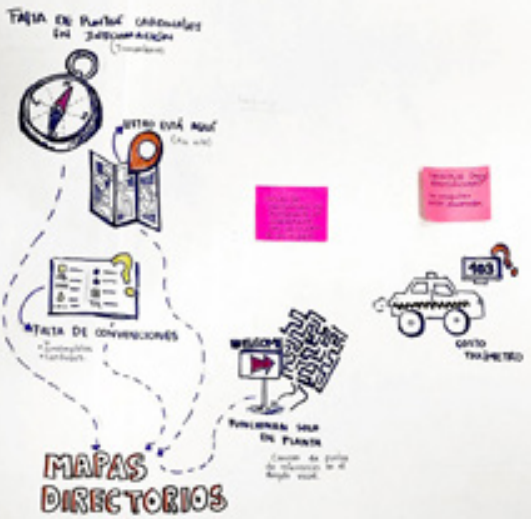




\section{Exploración de métodos y técnicas para la observación, recolección y procesamiento de la información ¿Cómo?}

De manera preliminar, se esbozan tres ámbitos de observación:

- Observación de comportamiento de usuarios frente a las problemáticas enunciadas.

- Observación de procesos y contraste con el contenido de los artefactos de información sobre los mismos, si existen.

- Observación de los artefactos de información disponibles, así como los hábitos de uso de los mismos.

Para estas observaciones, se inicia la exploración de métodos y técnicas disponibles en toolkits de diferentes autores y organizaciones que se interesan en investigación para el diseño centrado en el usuario (HCD, por sus siglas en inglés), tales como: Ideo.org ${ }^{1}$, The Digital Society School 2 , System Oriented Design ${ }^{3}$, Usability.gov 4 , y herramientas para el diseño centrado en el usuario (Agudelo \& Lleras, 2015). Todas estas son técnicas y herramientas basadas en el design thinking (pensamiento de diseño), que apoyadas en el uso creativo del lenguaje visual, detonan los procesos de generación de ideas, investigación, documentación, generación de prototipos y testeo en interacción con el usuario, en el marco de una permanente colaboración y comunicación entre todos los roles que participan en la investigación (véase figura 3).

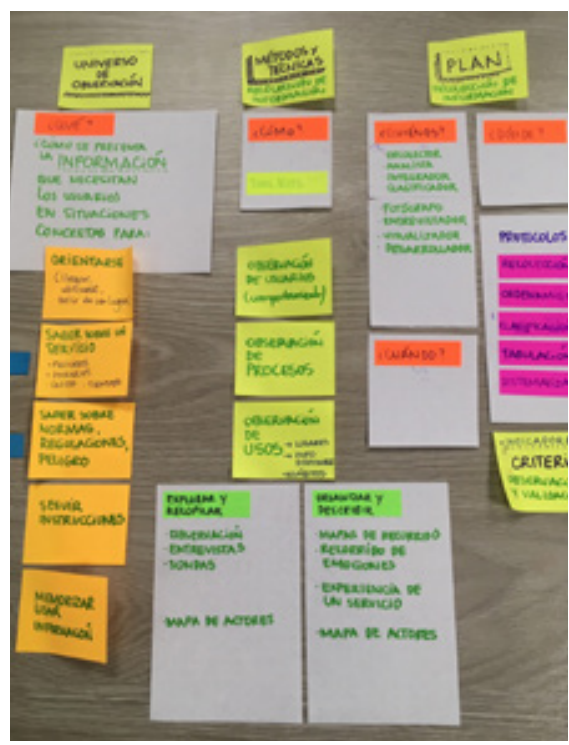

Figura 3. Mapeo preliminar de toolkits para la recolección de información Fuente: elaboración propia.

1Información relacionada en: https://www.designkit.org/methods

2 Información relacionada en: https://toolkits.dss.cloud/design/

3 Información relacionada en: https://www.systemsorienteddesign.net/index.php/tools

4 Información relacionada en: https://www.usability.gov/how-to-and-tools/methods/card-sorting.html 


\section{- 3. Diseño del plan de recolección para trabajo de campo: ¿Quiénes? ¿Dónde? ¿Cuándo?}

Esta fase se encuentra en prospectiva y en ella deben definirse los protocolos de recolección, ordenamiento, clasificación y tabulación de la información para su posterior sistematización. Estos deben considerar qué hacer antes, durante y después de cada proceso, así como los objetivos en cada caso, el foco y los recursos humanos, físicos, de desplazamiento, de registro y demás a lugar, para llevar a cabo el trabajo de campo.

En cuanto a los roles para el desarrollo de la observación y recolección de información, se prevén los siguientes: observador-recolector, fotógrafo, entrevistador, analista, clasificador, visualizador, integrador, desarrollador y quien a futuro gestione la difusión de los hallazgos y reportes.

Es deseable que quienes participen en las actividades del observatorio, sean estudiantes de diseño, diseñadores profesionales, investigadores en diseño, profesionales de otras disciplinas, funcionarios públicos, funcionarios del sector privado y ciudadanos usuarios de las categorías definidas en el universo de observación.

Al considerar que el proyecto de investigación cobra vida en la dinámica cambiante de un semillero de investigación, el dónde depende de los participantes en la siguiente convocatoria del semillero, en la que se definirá la delimitación geográfica o tipológica del universo de observación que tiene punto de inicio en la ciudad de Bogotá D.C., y que proyecta, en una etapa posterior, la vinculación de otras ciudades.

\section{- 4. Generación del sistema de información: ¿Cómo sistematizar la información recogida?}

El diseño del sistema de información del observatorio OPDI, se contempla a través de cuatro actividades básicas:

a. Entrada de la información.

b. Almacenamiento de la información.

c. Procesamiento de la información.

d. Salida de información (reportes y publicaciones).

Ya que se trata de una estrategia colaborativa para estimular el flujo de la información, se estima pertinente, como uno de los criterios orientadores, el planteamiento de Angulo (2009), quien proyecta dos vertientes: i) en formato digital que se apoye en herramientas de gestión del conocimiento, como bases de datos, lecciones aprendidas, buenas prácticas, minería de textos, además de procesos de monitoreo de información; y ii) articulando el contacto personal de los portadores del conocimiento, mediante herramientas presenciales o remotas, para el análisis colegiado de los hallazgos y la identificación de potenciales. 


\section{Resultados}

Entender la ciudad como un sistema interactivo en el que se teje una estructura de posibilidades de relación con los entornos, las interfaces, las dinámicas de servicios y los artefactos, llama al diseño a revisar su responsabilidad en la mediación que la haga más inteligible en los diversos niveles y escalas. La iniciativa del Observatorio en Problemáticas de Diseño de Información (OPDI) en el entorno ciudad, a la fecha ha mapeado las etapas para su desarrollo y ha delimitado las categorías de observación, con la utilización de métodos y técnicas de investigación en diseño centrado en el usuario (véase figura 4).

La exploración en cada una de las etapas, está procurando convocar e involucrar a más participantes, idealmente de otras instituciones académicas, ciudades e intereses, de la industria, del sector productivo y del sector público, para conformar una red que comparta este interés y generar un sistema de información que se sincronice con los desafíos de los enfoques emergentes del diseño, que forme para la comprensión de la complejidad, para la evaluación en diseño y el desarrollo de heurísticas pertinentes, para modular la pertinencia del diseño y la visualización de información, asi como la apropiación tecnológica, de manera que permita al diseño aprovechar su potencial de transformación en beneficio de las personas.

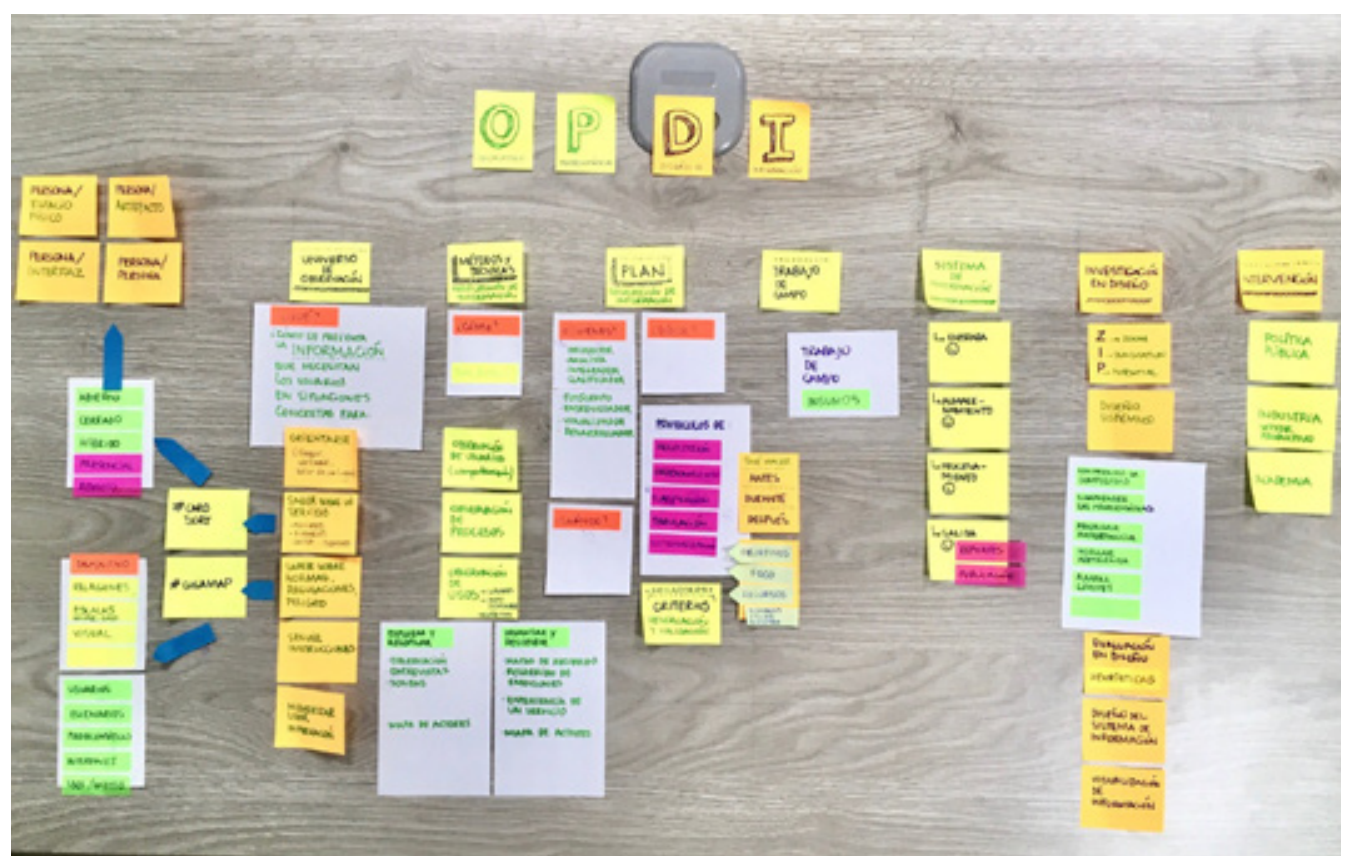

Figura 4. Mapeo en prospectiva del Observatorio en Problemáticas de Diseño de Información (OPDI).

Fuente: elaboración propia 


\section{Citas y referencias}

Agudelo Álvarez, N. L. \& Lleras, S. (2015). Para el salón: herramientas para el diseño centrado en el usuario. Bogotá, DC.: Ediciones Universidad de los Andes.

Angulo, N. (abril-junio, 2009) ¿Qué son los observatorios y cuáles son sus funciones? Innovación Educativa, 9(47) 5-17. Recuperado de https://www.redalyc.org/ pdf/1794/179414895002.pdf

Digital Society School. (s.f.). Design Method Toolkit [Contenido en un sitio web]. Recuperado de https://toolkits.dss.cloud/design/

Frascara, J. (2011) ¿Qué es el diseño de información? Buenos Aires: Ediciones Infinito.

Ideo.org. (s.f.). Design Kit [Contenido en un sitio web]. Recuperado de https://www.designkit. org/methods

Laroia, M.; Arun Kumar, P.; Minz, M.; Khateeb, B. \& Nahar, P. (2018). A case study on access to electricity. In: C.L. Remondino, B. Stabellini \& P. Tamborrini (Eds.), Visualizing Complex Systems. Catalogue of the RSD7 exhibition (pp. 67-69). Turin, Italy: Politécnico di Torino.

Pellegrini Filho, A. (2004). Presentación. En OPS (Ed.), La inteligencia colectiva: por una antropología del ciberespacio (pp.7-8; Trad. F. Martínez Álvarez). Recuperado de http://inteligenciacolectiva.bvsalud.org/channel.php?channel=1\&content=5

Sevaldson, B. (2018). Visualizing Complex Design: The Evolution of Gigamaps. In P. Jones \& K. Kijima (Eds.), Systemic Design (pp. 243-269). Tokyo: Springer.

Sevaldson, B. (June, 2011). GIGA-Mapping: Visualisation for complexity and systems thinking in design. In Nordic Design Research Conference, At Helsinki. Recuperado de https:// www.researchgate.net/publication/319930894_Giga-mapping_Visualisation_for_ Complexity_and_systems_thinking_in_design

System Oriented Design. (s.f.). Tools [Contenido en un sitio web]. Recuperado de https:// www.systemsorienteddesign.net

Usability.gov. (s.f.). Card sorting [Contenido en un sitio web]. Recuperado de https://www. usability.gov/how-to-and-tools/methods/card-sorting.html

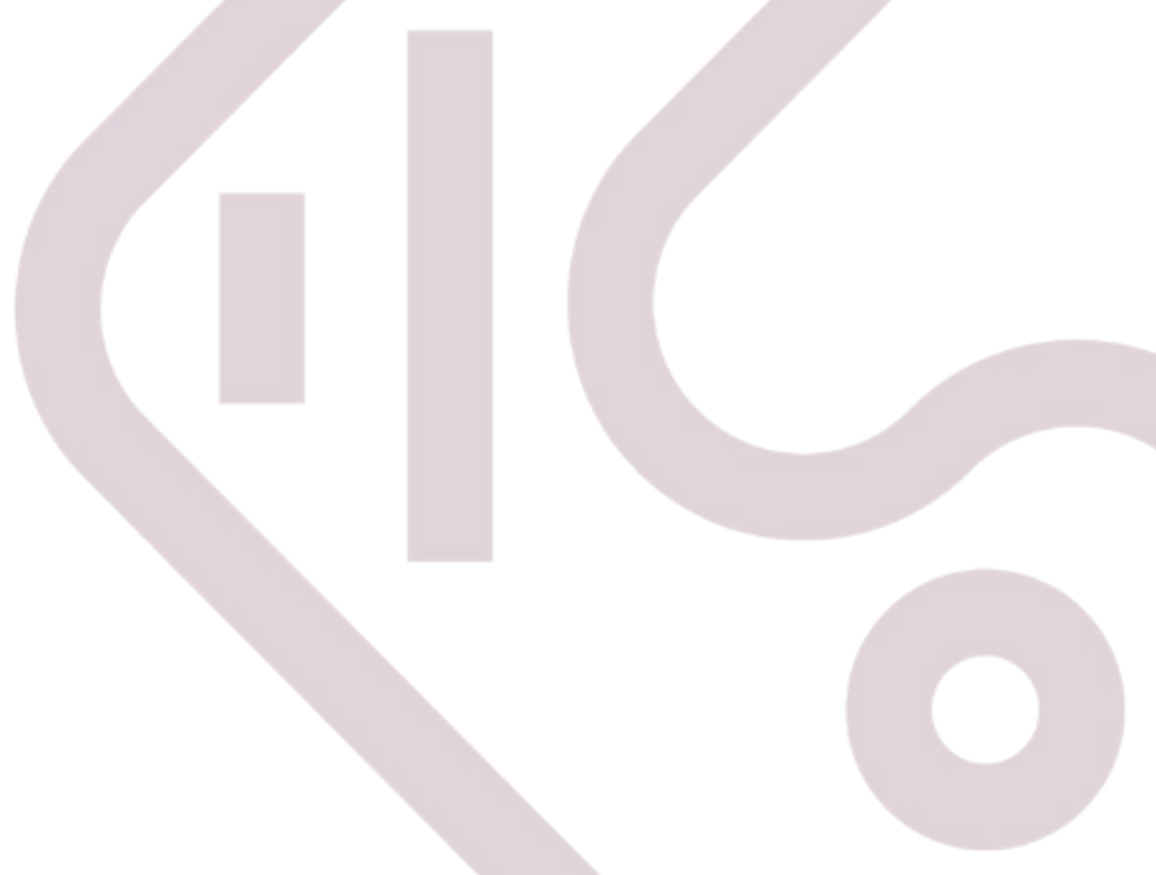

